\title{
Generalizations of a Matrix Inequality
}

\author{
Lingzhi Zhao ${ }^{1}$, Jun Yuan ${ }^{2}$, Yunfeng Cai ${ }^{3}$ \\ ${ }^{1}$ School of Mathematics and Information Technology, Nanjing Xiaozhuang University, Nanjing \\ ${ }^{2}$ College of Teacher Education, Nanjing Xiaozhuang University, Nanjing \\ ${ }^{3}$ College of Science, Nanjing University of Posts and Telecommunications, Nanjing \\ Email: yuanjun_math@126.com
}

Received June 16, 2013; revised July 16, 2013; accepted July 23, 2013

Copyright (C) 2014 Lingzhi Zhao et al. This is an open access article distributed under the Creative Commons Attribution License, which permits unrestricted use, distribution, and reproduction in any medium, provided the original work is properly cited. In accordance of the Creative Commons Attribution License all Copyrights (C) 2014 are reserved for SCIRP and the owner of the intellectual property Lingzhi Zhao et al. All Copyright (C) 2014 are guarded by law and by SCIRP as a guardian.

\section{ABSTRACT}

In this paper, some new generalizations of the matrix form of the Brunn-Minkowski inequality are presented.

\section{KEYWORDS}

\section{Brunn-Minkowski Inequality; Positive Definite Matrix; Determinant Differences}

\section{Introduction}

The well-known Brunn-Minkowski inequality is one of the most important inequalities in geometry. There are many other interesting results related to the Brunn-Minkowski inequality (see [1-8]). The matrix form of the Brunn-Minkowski inequality (see [9,10]) asserts that if $A$ and $B$ are two positive definite matrices of order $n$ and $0<\lambda<1$, then

$$
|\lambda A+(1-\lambda) B|^{\frac{1}{n}} \geq \lambda|A|^{\frac{1}{n}}+(1-\lambda)|B|^{\frac{1}{n}},
$$

with equality if and only if $A=c B(c \geq 0)$, where $|A|$ denotes the determinant of $A$.

Let $\mathbb{R}^{n \times n}$ denote the set of $n \times n$ real symmetry matrices. Let $I_{n}$ denote $n \times n$ unit matrix. We use the notation $A>0(A \geq 0)$ if $A$ is a positive definite (positive semi-definite) matrix, and $A^{*}$ denotes the transpose of $A$. Let $A, B \in \mathbb{R}^{n \times n}$, then $A>B(A \geq B)$ if and only if $A-B>0(A-B \geq 0)$.

If $A \in \mathbb{R}^{n \times n}$, then there exists a unitary matrix $U$ such as

$$
A=U^{*}\left[\lambda_{1}, \cdots, \lambda_{n}\right] U
$$

where $\left[\lambda_{1}, \cdots, \lambda_{n}\right]$ is a diagonal matrix $\left(\lambda_{i} \delta_{i j}\right)$, and $\lambda_{1}, \cdots, \lambda_{n}$ are the eigenvalues of $A$, each appearing as its multiplicity. Assume now that $f\left(\lambda_{i}\right) \in \mathbb{R}$ is well defined. Then $f(A)$ may be defined by (see e.g. [11, p. 71] or [12, p. 90])

$$
f(A)=U^{*}\left[f\left(\lambda_{1}\right), \cdots, f\left(\lambda_{n}\right)\right] U
$$

In this paper, some new generalizations of the matrix form of the Brunn-Minkowski inequality are presented. One of our main results is the following theorem.

Theorem 1.1. Let $A, B$ be positive definite commuting matrix of order $n$ with eigenvalues in the interval I. If $f$ is a positive concave function on $I$ and $0<\lambda<1$, then

$$
|f(\lambda A+(1-\lambda) B)|^{\frac{1}{n}} \geq \lambda|f(A)|^{\frac{1}{n}}+(1-\lambda)|f(B)|^{\frac{1}{n}}
$$


with equality if and only if $f$ is linear and $f(A)=c f(B)(c \geq 0)$.

Let $A, B \in \mathbb{R}^{n \times n}$, if $A \geq B$. We can define the determinant differences function of $A$ and $B$ by

$$
D_{d}(A, B)=|A|-|B| .
$$

The following theorem gives another generalization of (1).

Theorem 1.2. Let $A, B$ be positive definite commuting matrix of order $n$ with eigenvalues in the interval $I$ and $1 \in I$. Let $f$ be a positive function on $I$ and $a$ and $b$ be two nonnegative real numbers such that

$$
f(A)>a f\left(I_{n}\right), f(B)>b f\left(I_{n}\right) .
$$

Then

$$
D_{d}\left(f(A)+f(B),(a+b) f\left(I_{n}\right)\right)^{\frac{1}{n}} \geq D_{d}\left(f(A), a f\left(I_{n}\right)\right)^{\frac{1}{n}}+D_{d}\left(f(B), b f\left(I_{n}\right)\right)^{\frac{1}{n}}
$$

with equality if and only if $a^{-1} f(A)=b^{-1} f(B)$.

Remark 1. Let $f(t)=t$ in Theorem 1.1 or let $f(t)=t$ and $a=b=0$ in Theorem 1.2. We can both obtain (1). Hence Theorem 1.1 and Theorem 1.2 are generalizations of (1).

\section{Proofs of Theorems}

To prove the theorems, we need the following lemmas:

Lemma 2.1. ([13], p.472) Let $A, B \in \mathbb{R}^{n \times n}, A>B>0$. Then

$$
|A|>|B| \text {. }
$$

Lemma 2.2. ([13], p.50) Let $A, B \in \mathbb{R}^{n \times n}, A>0, B>0$. If $A$ and $B$ are commute, then exists a unitary matrix $U$ such that

$$
U^{*} A U=\left[a_{1}, a_{2}, \cdots, a_{n}\right] \text { and } U^{*} B U=\left[b_{1}, b_{2}, \cdots, b_{n}\right] .
$$

Lemma 2.3. ([14], p.35) Let $x_{i} \geq 0, y_{i} \geq 0(i=1,2, \cdots, n)$. Then

$$
\left(\prod_{i=1}^{n} x_{i}\right)^{\frac{1}{n}}+\left(\prod_{i=1}^{n} y_{i}\right)^{\frac{1}{n}} \leq\left(\prod_{i=1}^{n}\left(x_{i}+y_{i}\right)\right)^{\frac{1}{n}},
$$

with equality if and only if $x_{i}=v y_{i}$, where $v$ is a constant.

This is a special case of Maclaurin's inequality.

Proof of Theorem 1.1.

Since $A$ and $B$ are commuted, by lemma 2.2, there exists a unitary matrix $U$ such that

$$
A=U^{*}\left[a_{1}, a_{2}, \cdots, a_{n}\right] U \text { and } B=U^{*}\left[b_{1}, b_{2}, \cdots, b_{n}\right] U \text {. }
$$

Hence,

$$
\lambda A+(1-\lambda) B=U^{*}\left[\lambda a_{1}+(1-\lambda) b_{1}, \lambda a_{2}+(1-\lambda) b_{2}, \cdots, \lambda a_{n}+(1-\lambda) b_{n}\right] U .
$$

By (2), we have

$$
\begin{aligned}
& f(A)=U^{*}\left[f\left(a_{1}\right), f\left(a_{2}\right), \cdots, f\left(a_{n}\right)\right] U, \\
& f(B)=U^{*}\left[f\left(b_{1}\right), f\left(b_{2}\right), \cdots, f\left(b_{n}\right)\right] U,
\end{aligned}
$$

and

$$
f(\lambda A+(1-\lambda) B)=U^{*}\left[f\left(\lambda a_{1}+(1-\lambda) b_{1}\right), f\left(\lambda a_{2}+(1-\lambda) b_{2}\right), f\left(\lambda a_{n}+(1-\lambda) b_{n}\right)\right] U .
$$

Since $f$ is a concave function, by lemma 2.3 , we get 


$$
\begin{aligned}
& |f(\lambda A+(1-\lambda) B)|^{\frac{1}{n}}=\left(\prod_{i=1}^{n} f\left(\lambda a_{i}+(1-\lambda) b_{i}\right)\right)^{\frac{1}{n}} \\
& \geq\left(\prod_{i=1}^{n}\left[\lambda f\left(a_{i}\right)+(1-\lambda) f\left(b_{i}\right)\right]\right)^{\frac{1}{n}} \\
& \geq \lambda\left(\prod_{i=1}^{n} f\left(a_{i}\right)\right)^{\frac{1}{n}}+(1-\lambda)\left(\prod_{i=1}^{n} f\left(b_{i}\right)\right)^{\frac{1}{n}} \\
& =\lambda|f(A)|^{\frac{1}{n}}+(1-\lambda)|f(B)|^{\frac{1}{n}} .
\end{aligned}
$$

Now we consider the conditions of equality holds. Since $f$ is a concave function, the equality of (5) holds if and only if $f$ is linear. By the equality of Lemma 2.3, the equality of (6) holds if and only if $f\left(a_{i}\right)=c f\left(b_{i}\right)$, which means $f(A)=c f(B)$. So the equality of (3) holds if and only if $f$ is linear and $f(A)=c f(B)(c \geq 0)$. This completes the proof of the Theorem 1.1.

Applying the arithmetic-geometric mean inequality to the right side of (3), we get the following corollary.

Corollary 2.4. Let $A, B$ be positive definite commuting matrix of order $n$ with eigenvalues in the interval I. If $f$ is a positive concave function on $I$ and $0<\lambda<1$, then

$$
|f(\lambda A+(1-\lambda) B)| \geq|f(A)|^{\lambda}|f(B)|^{1-\lambda},
$$

with equality if and only if $A=B$.

Taking for $f(t)=t$ in Corollary 2.4, we obtain the Fan Ky concave theorem.

Proof of Theorem 1.2.

As in the proof of Theorem 1.1, since $A$ and $B$ are commuted, by lemma 2.2, there exists a unitary matrix $U$ such that

$$
f(A)=U^{*}\left[f\left(a_{1}\right), f\left(a_{2}\right), \cdots, f\left(a_{n}\right)\right] U
$$

and

$$
f(B)=U^{*}\left[f\left(b_{1}\right), f\left(b_{2}\right), \cdots, f\left(b_{n}\right)\right] U
$$

So

$$
\begin{aligned}
& |f(A)|=\prod_{i=1}^{n} f\left(a_{i}\right), \quad|f(B)|=\prod_{i=1}^{n} f\left(b_{i}\right), \\
& |f(A)+f(B)|=\prod_{i=1}^{n}\left(f\left(a_{i}\right)+f\left(b_{i}\right)\right) .
\end{aligned}
$$

It is easy to see that (4) holds if and only if

$$
\begin{aligned}
& \left(\prod_{i=1}^{n}\left(f\left(a_{i}\right)+f\left(b_{i}\right)\right)-[(a+b) f(1)]^{n}\right)^{\frac{1}{n}} \\
& \geq\left(\prod_{i=1}^{n} f\left(a_{i}\right)-[a f(1)]^{n}\right)^{\frac{1}{n}}+\left(\prod_{i=1}^{n} f\left(b_{i}\right)-[b f(1)]^{n}\right)^{\frac{1}{n}} .
\end{aligned}
$$

Since $f(A)>a f\left(I_{n}\right), f(B)>b f\left(I_{n}\right)$, by Lemma 2.1, we have

$$
\prod_{i=1}^{n} f\left(a_{i}\right)>[a f(1)]^{n}, \quad \prod_{i=1}^{n} f\left(b_{i}\right)>[b f(1)]^{n} .
$$

Now we prove (7). Put 


$$
X^{n}=\prod_{i=1}^{n} f\left(a_{i}\right)-[a f(1)]^{n}, \quad Y^{n}=\prod_{i=1}^{n} f\left(b_{i}\right)-[b f(1)]^{n} .
$$

Then

$$
X^{n}+[a f(1)]^{n}=\prod_{i=1}^{n} f\left(a_{i}\right), \quad Y^{n}+[b f(1)]^{n}=\prod_{i=1}^{n} f\left(b_{i}\right) .
$$

Applying Minkowski inequality, we have

$$
\begin{aligned}
\left((X+Y)^{n}+[(a+b) f(1)]^{n}\right)^{\frac{1}{n}} & \leq\left(X^{n}+[a f(1)]^{n}\right)^{\frac{1}{n}}+\left(Y^{n}+[b f(1)]^{n}\right)^{\frac{1}{n}} \\
& =\left(\prod_{i=1}^{n} f\left(a_{i}\right)\right)^{\frac{1}{n}}+\left(\prod_{i=1}^{n} f\left(b_{i}\right)\right)^{\frac{1}{n}}
\end{aligned}
$$

Using the Lemma 2.3 to the right of the above inequlity, we obtain

$$
\left((X+Y)^{n}+[(a+b) f(1)]^{n}\right)^{\frac{1}{n}} \leq\left(\prod_{i=1}^{n}\left(f\left(a_{i}\right)+f\left(b_{i}\right)\right)\right)^{\frac{1}{n}},
$$

which implies that

$$
(X+Y)^{n} \leq \prod_{i=1}^{n}\left(f\left(a_{i}\right)+f\left(b_{i}\right)\right)-[(a+b) f(1)]^{n} .
$$

It follows that

$$
X+Y \leq\left(\prod_{i=1}^{n}\left(f\left(a_{i}\right)+f\left(b_{i}\right)\right)-[(a+b) f(1)]^{n}\right)^{\frac{1}{n}},
$$

which is just the inequality (7).

By the equality conditions of Minkowski inequality and Lemma 2.3, the equality (1.4) holds if and only if $a^{-1} f\left(a_{i}\right)=b^{-1} f\left(b_{i}\right)$, which means $a^{-1} f(A)=b^{-1} f(B)$. Thus we complete the proof of Theorem 1.2.

Taking for $f(t)=t$ in Theorem 1.2, we obtain the following corollary.

Corollary 2.5. [7] Let $A, B$ be positive definite commuting matrix of order $n$ and $a$ and $b$ be two nonnegative real numbers such that

$$
A>a I_{n}, B>b I_{n} .
$$

Then

$$
\left(|A+B|-\left|(a+b) I_{n}\right|\right)^{\frac{1}{n}} \geq\left(|A|-\left|a I_{n}\right|\right)^{\frac{1}{n}}+\left(|B|-\left|b I_{n}\right|\right)^{\frac{1}{n}},
$$

with equality if and only if $a^{-1} A=b^{-1} B$.

\section{Acknowledgements}

The authors are most grateful to the referee for his valuable suggestions. And the authors would like to acknowledge the support from the National Natural Science Foundation of China (11101216,11161024), Qing Lan Project and the Nanjing Xiaozhuang University (2010KYQN24, 2010KYYB13).

\section{REFERENCES}

[1] I. J. Bakelman, “Convex Analysis and Nonlinear Geometric Elliptic Equations,” Springer, Berlin, 1994. http://dx.doi.org/10.1007/978-3-642-69881-1

[2] C. Borell, “The Brunn-Minkowski Inequality in Gauss Space,” Inventiones Mathematicae, Vol. 30, No. 2, 1975 , pp. $202-216$. http://dx.doi.org/10.1007/BF01425510 
[3] C. Borell, “Capacitary Inequality of the Brunn-Minkowski Inequality Type,” Mathematische Annalen, Vol. 263, No. 2, 1993, pp. 179-184. http://dx.doi.org/10.1007/BF01456879

[4] K. Fan, "Some Inequality Concerning Positive-Denite Hermitian Matrices," Mathematical Proceedings of the Cambridge Philosophical Society, Vol. 51, No. 3, 1958, pp. 414-421. http://dx.doi.org/10.1017/S0305004100030413

[5] R. J. Gardner and P. Gronchi, “A Brunn-Minkowski Inequality for the Integer Lattice,” Transactions of the American Mathematical Society, Vol. 353, No. 10, 2001, pp. 3995-4024. http://dx.doi.org/10.1090/S0002-9947-01-02763-5

[6] R. J. Gardner, “The Brunn-Minkowski Inequality,” Bulletin of the American Mathematical Society, Vol. 39, No. 3, 2002, pp. 355-405. http://dx.doi.org/10.1090/S0273-0979-02-00941-2

[7] G. S. Leng, “The Brunn-Minkowski Inequality for Volume Differences,” Advances in Applied Mathematics, Vol. 32, No. 3, 2004, pp. 615-624. http://dx.doi.org/10.1016/S0196-8858(03)00095-2

[8] R. Osserman, “The Brunn-Minkowski Inequality for Multiplictities,” Inventiones Mathematicae, Vol. 125, No. 3, 1996, pp. 405-411. http://dx.doi.org/10.1007/s002220050081

[9] E. V. Haynesworth, "Note on Bounds for Certain Determinants,” Duke Mathematical Journal, Vol. 24, No. 3, 1957, pp. 313320. http://dx.doi.org/10.1215/S0012-7094-57-02437-7

[10] E. V. Haynesworth, "Bounds for Determinants with Positive Diagonals," Transactions of the American Mathematical Society, Vol. 96, No. 3, 1960, pp. 395-413. http://dx.doi.org/10.1090/S0002-9947-1960-0120242-1

[11] M. Marcus and H. Minc, “A Survey of Matrix Theory and Inequalities,” Allyn and Bacon, Boston, 1964.

[12] R. Bellman, “Introduction to Matrix Analysis,” McGraw-Hill, New York, 1960.

[13] R. Horn and C. R. Johnson, “Matrix Analysis,” Cambridge University Press, New York, 1985. http://dx.doi.org/10.1017/CBO9780511810817

[14] E. F. Beckenbach and R. Bellman, “Inequalities,” Springer, Berlin, 1961. http://dx.doi.org/10.1007/978-3-642-64971-4 